\title{
Über das Balanophorin.
}

\author{
(I. Mitteilung) \\ von \\ M. Simon. \\ Aus dem chemischen Institut der k. k. Universität in Innsbruck.
}

(Vorgelegt in der Sitzung am 3. November 1910.)

Das Balanophorin ist ein Pflanzenstoff, der in den Balanophoren vorkommt, z. B. in Balanophora elongata, Balanophora globosa etc. Diese Schmarotzerpflanzen werden häufig in Java angetroffen.

Die Anregung zu dieser Arbeit gab mir Herr Prof. Heinricher, hier. Er war es auch, der mir in liebenswürdiger Weise durch Herrn Prof. M. Treub, Chef des Agrikulturdepartements zu Buitenzorg auf Java, das nötige Material versorgte. Ich spreche beiden Herren hiermit meinen besten Dank für ihre Bemühungen aus. ${ }^{1}$

\section{Literatur.}

Hauptsächlich in botanischer Beziehung wurde über die Balanophoren gearbeitet. Zuerst schrieb Göppert 1841 über den Bau der Balanophoren sowie über das Vorkommen von Wachs in ihnen (Nov. Act. acad. Caes. Leop.-Carol. Naturae Curiosorum vol. XVIII. Supplem. I.). Dann erschien im Jahre 1847 eine zweite Abhandlung von ihm zur Kenntnis der Balanophoren (1. c., tom. XXII). Später im Jahre 1869 hat Beccari sich damit befaßt; er schrieb: „Illustrazione di nuove specie di piante Bornensi. Balanophorae (Nuovo Giornale Botanic. Italian., Vol. I). In letzter Zeit wurde dieses Thema in ausführlicher Weise von Herrn Prof. Heinricher und unter

1 Prof. Tre ub ist leider am 3. Oktober 1. J. verstorben. 
seiner Anleitung von seinem Assistenten Herrn Dr. Strigl bearbeitet. Es erschien von Heinricher $»$ Beiträge zur Kenntnis der Gattung Balanophora (Sitzungsber, d. kais. Akad. d. Wissenschaften in Wien, mathem.-naturw. Klasse, Bd. 116) und »Van Tieghem's Anschauungen über den Bau der Balanophorenknolle« (1. c., Bd.117). Strig1 lieferte: Der anatomische Bau der Knollenrinde von Balanophora (1. c., Bd. 116) und »Der Thallus von Balanophora (1. c., Bd. 117).

In chemischer Hinsicht ist wohl nur die Arbeit Theodor Poleck's von Bedeutung; er schrieb über die chemische Untersuchung des wachsähnlichen Bestandteiles der Balanophora elongata (Nov. Act, acad. Caes. Leop.-Carol., Naturae Curiosorum tom. XXII, 1847). Poleck berichtet darin, daß diese Parasitengattung von den Einheimischen geradezu als Kerze verwendet wird. Göppert gab 1841 den Namen Balanophorin für die wachsähnliche Substanz der Balanophoren. Dieselbe ist in Äther löslich und kann damit ausgezogen werden. Im Wasserbad erhitzt, wird das Wachs oder Harz durchsichtig und gelblich. Die Substanz ist etwas klebrig und hat schwachen balsamischen Geruch. Sie wurde auch mit Wasser destilliert, wobei das Destillat den Geruch des Harzes annahm. Unlöslich in Wasser, sehr wenig in wässerigem Alkohol, wird das Harz in größerer Menge von absolutem, Alkohol aufgenommen, scheidet sich aber beim Abkühlen der erwärmten Lösung wieder aus. Auch in ätherischen und fetten Ölen sowie in Schwefelkohlenstoff löst sich die Substanz. In Ammoniak unlöslich, ebenso in verdünnter Kalilauge, wird das Harz auch von konzentrierter Lauge beim Kochen nicht zersetzt. In konzentrierter Schwefelsäure löst es sich in der Wärme und kann durch Wasser wieder gefällt werden; Vitriolöl verkohlt es bei längerem Erhitzen. Poleck machte auch mit der durch Äther gereinigten Substanz Verbrennungsanalysen und fand in zwei Proben einmal $79 \cdot 46 \%$ Kohlenstoff und $11 \cdot 28 \%$ Wasserstoff und dann $79 \cdot 72 \%$ Kohlenstoff und 11:20\% Wasserstoff. Er berechnet daraus für die Substanz die Formel $\mathrm{C}_{12} \mathrm{H}_{20} \mathrm{O}$, welche $80.00 \%$ Kohlenstoff und $11.11 \%$ Wasserstoff verlangt. Poleck untersuchte weiter das Verhalten der Substanz zur Salpetersäure, konnte aber wegen Mangel an Material die 
erhaltenen Produkte nicht vollständig prüfen. Zum Schluß spricht er die Substanz als Harz an und vergleicht sie mit Analysen anderer Harze wie Euphorbium, Mastix, Elemi, Bienenwachs und Japanwachs, die ähnliche Prozentzahlen von Kohlenstoff und Wasserstoff zeigen.

Erwähnt möge noch werden, daß Göppert (a. a. O.) den ungemeinen Reichtum an der wachsartigen Substanz in den untersuchten Balanophoren besonders hervorhebt.

\section{Herstellung des Rohbalanophorins und Reinigungsversuche desselben.}

Das Material kam direkt von Java in einem verlöteten Blechkasten, worin die Knollen in Alkohol eingelegt waren. Der Kasten befand sich zum Schutze noch in einer starken Holzkiste.

Die Knollen wurden von anhängender Erde durch $\mathrm{Ab}$ bürsten möglichst befreit und darauf in dünne Scheiben geschnitten, um sie dann an der Luft trocknen zu lassen. Die geschnittene getrocknete Menge betrug $1560 \mathrm{~g}$. Die Scheiben wurden nun zerkleinert und darnach der Extraktion unterworfen.

Als Extraktionsapparat wurde ein solcher von Guérin mit geringen Abänderungen benützt. Abbildung und Beschreibung davon findet sich in der Zeitschrift für analytische Chemie, Bd. 19, 472, oder auch Zentralblatt, 3. Folge, 11, 189 (1880).

Als Extraktionsmittel diente frisch destillierter. Äther. Da das Material sehr reich an Balanophorin ist, wurde das grobe Pulver erst in einem großen Kochkolben zweimal mit Äther (am Rückflußkühler) ausgekocht, dann nach Filtration des Extraktes der noch an dem Pulver haftende Äther abdestilliert und das Material darauf in den Apparat gebracht. Bei verschlossenem Abflußrohr wurde nun der Äther einen halben bis einen Tag darauf einwirken gelassen und, nachdem dann der Äther in den Siedeballon abgelassen war, mit der Extraktion in der Wärme begonnen. Diese nahm einen Tag in Anspruch und zur Aufarbeitung des Materials konnte der Apparat fünfmal 
beschickt werden. Die ätherischen Lösungen wurden in einem größeren Erlenmeyerkolben gebracht und vom Äther durch Destillation so weit wie möglich befreit. Die Rückstände wurden dann in einer Porzellanschale gesammelt und am Wasserbade erwärmt, um die Ätherreste zu entfernen. Die Ausbeute an Rohbalanophorin betrug $1010 \mathrm{~g}$; das sind rund $65 \%$.

Die Rohsubstanz bildet nach dem Erkalten eine graubraune Masse. Die Farbe rührt her von beigemengten erdigen. und pflanzlichen Teilchen. Um dieselben wegzubringen, wurde der Rohstoff filtriert. Dazu wurde ein Heißwassertrichter verwendet, der mit einem Rückflußkühler versehen war und Tag und Nacht sich im Gange befand. Die abfließende Substanz wurde in kleinen Porzellanschalen aufgefangen. Das Filtrieren ging sehr langsam; es dauerte vier Monate.

Die filtrierte Substanz ist klar und von honiggelber Farbe, erstarrt langsam, wobei sie ein wachsähnliches Aussehen annimmt, und ist in der Kälte spröde. Durch die Handwärme wird sie sehr bald weich und klebrig und zieht dann Fäden so dünn wie Seide.

Zur Reinigung wurden $23 \mathrm{~g}$ Rohsubstanz mit $400 \mathrm{~cm}^{3}$ Alkohol am Rückflußkühler gekocht, dann vom Reste abfiltriert und zur Krystallisation in den Eiskasten gestellt. Der Rest wurde wieder mit $400 \mathrm{~cm}^{3}$ Alkohol behandelt und im ganzen wurden $3200 \mathrm{~cm}^{3}$ Alkohol bis zur völligen Lösung benötigt; ein Teil der Substanz löst sich somit in etwa 140 Teilen Alkohol von beiläufig $95 \%$.

Die Menge der gereinigten Substanz betrug $17 \mathrm{~g}$. Diese wurde nun in Aceton am Wasserbad gelöst, filtriert und wieder in den Eiskasten zum Abkühlen gestellt. Zur Lösung wurden im ganzen $850 \mathrm{~cm}^{3}$ Aceton gebraucht; die Löslichkeit ist hier also etwa $1: 50$.

Die abgeschiedene Substanz zeigt nach dem Absaugen und Trocknen ein weißes Pulver, welches aber auch unter dem Mikroskope keine deutlichen Krystalle erkennen läßt, sondern nur Körner bildet. Auch Versuche mit recht langsamem Abkühlenlassen hatten keinen besseren Erfolg.

Eine genaue qualitative Prüfung dieser Substanz zeigte, daß sie nur Kohlenstoff und Wasserstoff enthält, $z u$ denen 
noch als Ergebnis der Elementaranalyse (aus dem Defizit von $100 \%$ ) sich Sauerstoff gesellt.

Zwei Verbrennungsanalysen des soweit gereinigten Stoffes mögen angeführt sein:

I. $0.2923 \mathrm{~g}$ Substanz gaben $0.8430 \mathrm{~g} \mathrm{CO}_{2}$ und $0.2841 \mathrm{~g} \mathrm{H}_{2} \mathrm{O}$.

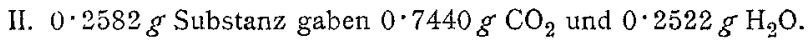

In 100 Teilen gefunden:

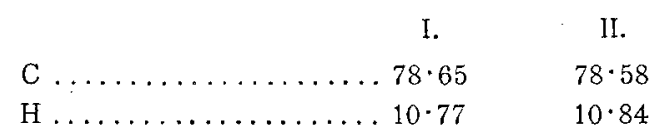

Hierauf wurde die Substanz noch zweimal aus Aceton umkrystallisiert und eine Elementaranalyse gemacht (I) und nach weiterer dreimaliger Krystallisation wurde eine zweite Verbrennung ausgeführt (II). Die Resultate sind die nachfolgenden:

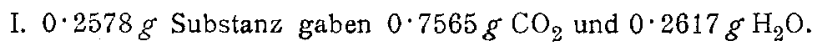

II. $0.2733 \mathrm{~g}$ Substanz gaben $0.7994 \mathrm{~g} \mathrm{CO}_{2}$ und $0.2785 \mathrm{~g} \mathrm{H}_{2} \mathrm{O}$.

In 100 Teilen:

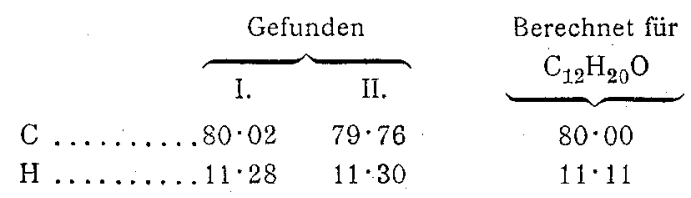

Diese Analysen stimmen mit den von Th. Poleck angegebenen (siehe oben) sehr gut überein.

Außer den von Poleck angegebenen Lösungsmitteln für das Balanophorin sind noch zu nennen: Petroleumäther, Benzin, Ligroin, Chloroform, Tetrachlorkohlenstoff und Benzol.

Als Reinigungsversuch wurde auch eine Destillation im Dampfstrom duichgeführt. $Z \mathrm{u}$ dem $Z$ weck wurden zirka $6 g$ Substanz durch 6 Stunden im Dampfstrom destilliert. Es wurden dabei sechs Kolben voll Destillat aufgefangen; die Kolben hatten einen Inhalt von 250 bis $400 \mathrm{~cm}^{3}$. Dieses Destillat war trüb, hatte einen eigenen Geruch und schied später einzelne kleine Flocken ab; von der Substanz ging also hierbei nichts Nennenswertes über. Der Rückstand im Siede- 
kolben wurde durch Absaugen des Wasserdampfes unter Erwärmen auf dem Wasserbade getrocknet und dann dreimal aus Aceton umkrystallisiert.

Die Substanz ist dann, wie aus der Verbrennung hervorgeht, von der gleichen Reinheit, wie selbe durch Alkohol- und dreimalige Acetonkrystallisation erbalten wurde.

$0.2577 g$ Substanz gaben $0.7441 g \mathrm{CO}_{2}$ und $0.2552 \mathrm{~g} \mathrm{H}_{2} \mathrm{O}$.

In 100 Teilen gefunden:

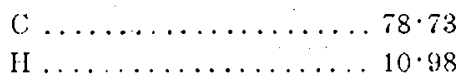

Der Schmelzpunkt des reinen Balanophorins liegt zwischen 56 bis $57^{\circ} \mathrm{C}$. Dabei bildet es eine wasserklar farblose Flüssigkeit von der Konsistenz des Glycerins, die erst allmählich unter Trübwerden erstart, um schließlich wie weißes Wachs zu erscheinen.

Bci höhercm Erhitzen brennt dieser Stoff, wie schon erwähnt, mit helleuchtender Flamme und verbreitet dabei einen eigentümlichen Geruch, der mit Akroleindämpfen verwechselt werden kann. Um nun festzustellen, ob Akrolein dabei ist oder nicht, wurden die Dämpfe von stark erhitztem Balanophorin in fuchsinschweflige Säure geleitet. Diese wurde aber nicht rot gefärbt, auch bei einem zweiten Versuche nicht, wo zum Balanophorin etwas saures schwefelsaures Kali zugesetzt worden war. Auch gaben die in Wasser geleiteten Dämpfe mit ammoniakalischer Silberlösung keinen Silberspiegel. Es bildet sich somit beim Erhitzen von Balanophorin kein Akrolein.

\section{Versuche zur Zerlegung des Balanophorins.}

Wic Poleck bemerkt, wird die Substanz weder durch verdünnte noch konzentrierte Lauge zersetzt und von mir angestellte Versuche mit alkoholischer $1 / 10^{-}$und $n / 2$ - KHO ergaben weder eine Säurezahl, noch auch konnte cine Verseifungszahl erhalten werden. Ebenso konnte das Balanophorin mit konzentrierter. alkoholischer Kalilauge in einem zugeschmolzenen Glasrohr, welches stundenlang im Schießofen auf zirka $140^{\circ} \mathrm{C}$. erhitzt war, nicht zersetzt werden. 
Nach vielen anderen vergeblichen Versuchen gelang es endlich, auf zwei Wegen das Balanophorin zu zersetzen. Den einen Weg bildet die Destillation im luftverdünnten Raum, den zweiten die Kalischmelze.

Zur Destillation im Vakuum wurde der Fraktionskolben mit einem Thermometer und einer Kapillare versehen, durch welche gewaschene und getrocknete Kohlensäure zutrat. Als Vorlage diente eine solche nach Brüh!.

Zum crsten Versuche wurden etwa $30 \mathrm{~g}$ Rohbalanophorin verwendet. Dic Destillation der Substanz beginnt, nachdem noch Ätherreste unter Aufschäumen entwichen waren, bei $275^{\circ}$. Beim Steigen der Temperatur bis $300^{\circ}$ geht eine erst weiße, später gelbliche, krystallisch erstarrende Masse über. Destilliert man weiter, so folgt darauf cine dünnflüssige, leicht erstarrende Substanz, die einem Harze - etwa Kolophonium ähnelt; die Temperatur bleibt dabei $350^{\circ}$. Der Rest im Fraktionskolben ist dunkel gefärbt.

Der bei diesem Versuche bis zu $300^{\circ}$ übergégangene Teil wurde nochmals im Vakuum destillicrt. Die Hauptmenge dieser neuerlichen. Destillation ging bei 240 bis $250^{\circ}$ über und betrug $10 \mathrm{~g}$. Die erhaltene Substanz ist in Alkohol sehr leicht löslich und wurde daraus einmal umkrystallisiert, wobei $7 g$ schön krystallisiertes Produkt erhalten wurden. Die Krystalle sind glänzende Schuppen, welche dem Aussehen nach an Borsäure oder Naphthalin erinnern. In Kalilauge wie auch in Sodalösung ist diese Substanz leicht löslich und aus der Lösung durch eine Mineralsäure wieder fällbar; auch zeigen die Krystalle, auf Lackmuspapier gepreßt, schon saure Reaktion. Daher wurde das Produkt als eine Säure angesprochen.

Der höher übergegangene Teil der Zersetzung im luftverdünnten Raume wurde auch nochmals einer solchen Destillation unterworfen. Die Hauptmasse geht dabci zwischen 350 und $370^{\circ}$ über und bildet eine gelbe durchsichtige und spröde Substanz, welche leicht löslich in Äther, Chloroform und Essigäther ist. In Alkohol ist sie schwerer löslich. Behandelt man die Substanz unter Erwärmen mit alkoholischer Kalilauge, so gibt das Filtrat davon nach dem Ansäuern mit Salzsäure 
kaum eine Trübung; es kann also wohl von der oben erwähnten Säure nichts mehr enthalten sein.

Später wurde noch ein größerer Versuch mit einer solchen Destillation gemacht, wobei $91 \mathrm{~g}$ Rohbalanophorin in Verwendung kamen. Hier zeigte das Manometer $19 \mathrm{~mm}$ Druck. Das Sieden begann hier bei $242^{\circ}$, die' Säure ging bis $262^{\circ}$ fort $(31 \mathrm{~g})$, eine Mischung von Säure und harzartiger Substanz destillierte von 262 bis $324^{\circ}$ und fast reine harzartige Substanz floß dann bis zu $340^{\circ} \mathrm{ab}$. Die Dauer der ganzen Destillation betrug $8 \frac{1}{2}$ Stunden.

Um aus dem zweiten Teile der Destillation die Säure zu gewinnen, wurde die Masse mit Alkohol (am Rückflußkühler) erwärmt, dann abkühlen gelassen, wobei außer dem verbliebenen Reste der harzartigen Substanz noch etwas davon gelöste sich am Boden des Kolbens absetzt. Darauf wurde filtriert und das Filtrat stark eingeengt, wonach beim Abkühlen die Säure auskrystallisiert. Auf diese Weise wurden noch $13 \mathrm{~g}$ Säure gewonnen, während die harzartige Substanz $8 g$ wog. Der dritte Teil der Destillation ergab $29 \mathrm{~g}$ Harzsubstanz.

Die Säure von der ersten Fraktion ( $31 \mathrm{~g}$ ) wurde in einem Kolben mit Alkohol behandelt und lieferte bei der ersten Krystallisation $12 \mathrm{~g}$ reines Produkt in schönen blättrigen Krystallen. Unterwirft man diese reine Säure einer Destillation im Vakuum, so liegt ihr Siedepunkt bei $18.5 \mathrm{~mm}$ Druck konstant bei 226 bis $227^{\circ}$. Es gingen dabei $7.7 \mathrm{~g}$ über, welche nach dem Erstarren ganz das Aussehen von Stearin hatten. Im übrigen wurden noch aus der Mutterlauge von obiger Krystallisation und aus der von der zweiten Fraktion erhaltenen Säure im Vakuum 14.g reines Produkt gewonnen.

Von der Kalischmelze sollen folgende Versuche angeführt werden:

Nach einer Vorprobe (mit $2 \mathrm{~g}$ Substanz) wurden $20 \mathrm{~g}$ filtriertes Rohbalanophorin mit $70 \mathrm{~g}$ : Atzkali im Silbertiegel geschmolzen. Ein Thermometer mit Silberhülse diente zugleich als Rührer. Zuerst schwimmt das geschmolzene Balanophorin auf dem Kali, dann tritt eine Reaktion ein, wobei sich eine braune klumpige Masse bildet, welche sich vom überschüssigen Kali trennt (bei $150^{\circ}$ ). Erhitzt man höher, so tritt Schäumen 
ein (bei $210^{\circ}$ ), nach dem Abkühlen aber scheidet sich wieder die Masse vom Kali. Die erkaltete Schmelze wird in Wasser, welches mit Alkohol (etwa 1:10) versetzt ist, gebracht. Nach dem Erwärmen auf dem Wasserbad und erfolgter Abkühlung fügt man zur: Klärung Äther hinzu und gibt das Ganze in einen Scheidetrichter. Der Äther, welcher noch mit Wasser - nach Abfließenlassen der unteren Schicht - ausgeschüttelt wird, enthält die nicht sauren Bestandteile, während die Säure an Kalium gebunden im wässerigen Teil und dem Wasser, womit der Äther ausgeschüttelt wurde, verbleibt.

Um nun die Säure $z u$ gewinnen, wurde der wässerige Teil vereint mit dem Wasser, womit der Äther ausgeschüttelt wurde, auf dem Wasserbade bis zur Vertreibung der Alkohol- und Ätherreste erwärmt, dann in einen Kolben gespült und mit verdünnter Schwefelsäure angesäuert. Die ausgefallene Säure wurde abgesaugt, dann dreimal aus Alkohol krystallisiert und hierauf noch in verdünnter Kalilauge gelöst, mit Schwefelsäure gefällt und nochmals aus Alkohol krystallisiert. Diese Säure ist, wie später gezeigt werden wird, die gleiche wie die durch Vakuumdestillation erhaltene.

Von dem in Äther gelösten Teil der Zersetzung mit Kali wurde dieser abdestilliert, wobei eine weißliche Masse zurückbleibt, aus welcher mit Aceton eine krystallisierte Substanz erhaiten werden konnte. Der Rückstand aus Äther wog $10.5 \mathrm{~g}$ bei $20 \mathrm{~g}$ zersetztem Balanophorin.

Mit der Kalischmelze wurde noch ein Versuch in größerem Maßstabe gemacht, wozu $100 \mathrm{~g}$ Rohbalanophorin und $300 \mathrm{~g}$ Kalihydrat verwendet wurden. Zur Zersetzung des gebildeten Salzes wurde hierbei Salzsäure statt Schwefelsäure genommen. Infolge eines größeren Verlustes können die Gewichtsangaben über die erhaltenen Zersetzungsprodukte nur ganz beiläufige sein. Die gewonnene Säure betrug $47 \cdot 2 \mathrm{~g}$, woraus $16 \mathrm{~g}$ reines Produkt durch Alkoholkrystallisation erhalten wurden. Die in der Mutterlauge verbleibende Säure kann am besten durch Destillation im luftverdünnten Raume rein erhalten werden oder durch Überführen derselben in den Methylester und nachfolgende Verseifung. 
Aus dem Äther von diesem Versuche wurden $41.5 \mathrm{~g}$ des zweiten Zersetzungsproduktes vom Balanophorin erhalten.

\section{Von der aus dem Balanophorin erhaltenen Säure.}

Wie schon oben erwähnt, läßt sich die Säure sowonl aus den Produkten der Vakuumdestillation wie auch aus denjenigen der Kalischmelze schön krystallisiert erhalten. Als Lösungsmittel dazu diente der Alkohol; auch Benzol liefert schöne Krystallisationen.

Die Säure hat - auf beiden Wegen hergestellt - einen Schmelzpunkt von $62.5^{\circ}$ und siedet bei $18.5 \mathrm{~mm}$ Druck bei 226 bis $227^{\circ}$. Bei einer anderen Destillation, wo ein Druck von $55 \mathrm{~mm}$ erreicht wurde, lag der Siedepunkt bei 246 bis $248^{\circ}$.

Un zu sehen, ob es eine gesättigte oder ungesättigte Verbindung ist, wurde eine kleine Probe in Wasser suspendiert und mit einer verdünnten Lösung von Kaliumpermanganat und Soda geschüttelt. Die violette Farbe des Permanganats blieb aber bestehen, ebenso wie bei einer zweiten Probe, welche mit verdünntem Bromwasser versetzt war, die Gelbfärbung durch das Brom nicht verschwand. Es liegt also eine gesättigte Säure vor.

Von den nun folgenden Elementaranalysen möge eine hier angeführt werden:

$0.2506 \mathrm{~g}$ Substanz gaben $0.6708 \mathrm{~g} \mathrm{CO}_{2}$ und $0.2735 \mathrm{~g} \mathrm{H}_{2} \mathrm{O}$.

In 100 Teilen:

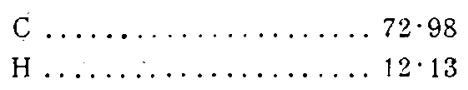

Diese und andere Analysen würden auf eine Tridecylsäule $\mathrm{C}_{13} \mathrm{H}_{26} \mathrm{O}_{2}$ schließen lassen, welche einer Berechnung von $72 \cdot 89 \%$ Kohlenstoff und $12 \cdot 14 \%$ Wasserstoff entspricht.

Als jedoch Titrationen mit der Säure vorgenommen wurden, wurde stets weniger Lauge verbraucht, als die Berechnung aufwies. Ebenso gaben die Bestimmungen der Basen in mit der Säure hergestellten Salzen Resultate, die nicht in Einklang zu bringen waren mit einer Tridecylsäure. 
Nach verschiedenen Versuchen wurden brauchbare Werte der Elementaranalyse erhalten, die mit den Titrationen und den Bestimmungen der Metalle in den Salzen zusammenstimmten, sobald die Substanz mit Kupferoxyd gemischt verbrannt wurde.

Die Säure wurde daraufhin als Palmitinsäure angesprochen. ${ }^{1}$ Die Analysen lieferten folgende kiesultate:

I. $0 \cdot 3106 g$ Substanz gaben $0 \cdot 8534 g \mathrm{CO}_{2}$ und $0.358 \mathrm{~s} g \mathrm{H}_{2} \mathrm{O}$.

II. $0.2038 \mathrm{~g}$ Substanz gaben $0.5591 \mathrm{~g} \mathrm{CO}_{2}$ und $0.2304 \mathrm{~g} \mathrm{H}_{2} \mathrm{O}$.

III. $0.1998 g$ Substanz gaben $0.5491 g\left(: \mathrm{O}_{2}\right.$ und $0.2258 g \mathrm{H}_{2} \mathrm{O}$.

In 100 Teilen:

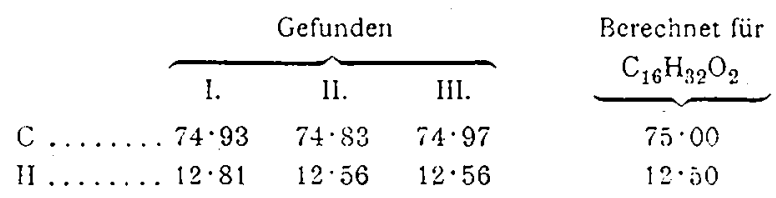

Weiters wurde eine Verbrennung von Säure ausgeführt, die bei der Kalischmelze gewonnen worden war.

$0.3422 g$ Substanz gaben $0.9403 g \mathrm{CO}_{2}$ und $0.4013 \% \mathrm{H}_{2} \mathrm{O}$.

In 100 Teilen:

$\begin{array}{ccc}\mathrm{C} \ldots \ldots \ldots \ldots & \underbrace{\text { Gefunden }}_{74 \cdot 94} & \underbrace{\begin{array}{c}\text { Berechnet für } \\ \mathrm{C}_{16} \mathrm{H}_{32} \mathrm{O}_{2}\end{array}}_{75 \cdot 00} \\ \mathrm{H} \ldots \ldots \ldots \ldots & 12 \cdot 73 & 12 \cdot 50\end{array}$

Da der Schmelzpunkt und auch die Elementaranalyse der Säure gleich ist, sei sie auf dem einen oder dem anderen Wege hergestellt, so ist es wohl die gleiche Substanz, die dabei erhalten wird.

Auch das Molekulargewicht wurde zu bestimmen versucht. Nach mehreren Versuchen konnte mit Chloroform noch am ehesten ein Resultat erreicht werden. ${ }^{2}$ Das Ergebnis dieser Bestimmung war das folgende:

1 Auch reine Palmitinsäure von der Firma Kahlbaum in Berlin lieferte, nur auf diese Weise verbrannt, brauchbare Werte.

2 Nach Beckmann's Methode. 
Konstante für Chloroform $=36$.

\begin{tabular}{|c|c|c|c|c|}
\hline $\begin{array}{c}\text { Menge des } \\
\text { Chloroforms }\end{array}$ & $\begin{array}{c}\text { Gewicht der } \\
\text { Substanz }\end{array}$ & $\begin{array}{c}\text { Beobachtete } \\
\text { Temperatur- } \\
\text { erhöhung }\end{array}$ & $\begin{array}{c}\text { Gefundenes } \\
\text { Molekular- } \\
\text { gewicht }\end{array}$ & $\begin{array}{c}\text { Berechnet } \\
\text { für } \\
\mathrm{C}_{16} \mathrm{H}_{32} \mathrm{O}_{2}\end{array}$ \\
\hline $27 \cdot 10$ & 0.3282 & 0.18 & 242 & \\
$27 \cdot 10$ & 0.7 .134 & 0.34 & 278 & 256 \\
\hline
\end{tabular}

Schließlich wurden noch einige Titrationen vorgenommen, welche gleichzeitig zur Bestätigung der Bestimmung des Molekulargewichtes dienen sollen. Als Resultat wurde erhalten:

I. $1.3030 \mathrm{~g}$ Substanz brauchten zur Neutralisation $0.2846 \mathrm{~g}$ Kalihydrat. Von einer Krystallisation aus Benzol.

II. $1 \cdot 2037 . g$ Substanz brauchten zur Neutralisation $0.2614 \mathrm{~g}$ Kalihydrat. Von einer zweiten Krystallisation aus Benzol.

III. $0.6018 \mathrm{~g}$ Substanz brauchten zur Neutralisation $0.1317 \mathrm{~g}$ Kalihydrat. Von einer Krystallisation aus Alkohol.

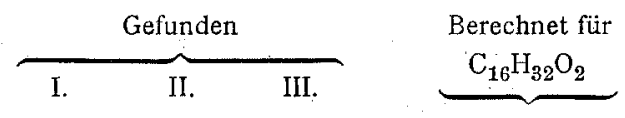

Verseifungszahl (Anzahl der Milligramme $\mathrm{KHO}$, die $1 \mathrm{~g}$ $\begin{array}{llll}\text { der Substanz neutralisieren) } 218 \cdot 4 & 217 \cdot 2 & 218 \cdot 8 & 218 \cdot 7\end{array}$

Der Schmelzpunkt von $62 \cdot 5^{\circ}$ stimmt mit den Angaben über den Schmelzpunkt der Palmitinsäure (62 und $62 \cdot 6^{\circ}$ ) gut überein. ${ }^{1}$ Ebenso sind die Siedepunkte von 226 bis $227^{\circ}$ bei $18.5 \mathrm{~mm}$ Druck und von 246 bis $248^{\circ}$ bei $55 \mathrm{~mm}$ Druck mit den folgenden Angaben: $215^{\circ}$ bei $15 \mathrm{~mm}$ und 268.5 bis $271.5^{\circ}$ bei $100 \mathrm{~mm}$ wohl in Einklang zu bringen. ${ }^{2}$

Es liegt also hier Palmitinsäure vor und zur weiteren Bestätigung für die Annahme derselben sollen noch die dargestellten Salze und einige Analysen derselben aufgeführt werden.

1 Beilstein, Handb. d. org. Chemie, 3. Aufl., I, 443 und 1. Ergänzungsband zu Beilstein's Handb., 159.

2 Ibidem. 


\section{Das Kalisalz.}

Selbes wurde hergestellt durch Neutralisation der Säure mit Kalilauge. Es bildet kleine Krystalle (I und II).

Weiters wurde das Salz gewonnen aus in Ätheralkohol gelöster Säure, die mit trockenem kohlensauren Kali versetzt war. Durch langes Erhitzen auf dem Wasserbade schied sich hierbei das Salz in schönen Krystallschuppen ab (III und IV).

Die Analysen ergaben:

I. $0 \cdot 2202 g$ Substanz gaben, mit Kupferoxyd und Kaliumbichromat verbrannt, $0.5279 \mathrm{~g} \mathrm{CO}_{2}$ und $0.2146 \mathrm{~g} \mathrm{H}_{2} \mathrm{O}$.

II. $0 \cdot 2939 \mathrm{~g}$ Substanz gaben nach dem Verbrennen $0.0690 \mathrm{~g} \mathrm{~K}_{2} \mathrm{CO}_{3}$.

III. $0.2653 \mathrm{~g}$ Substanz gaben $0.0612 \mathrm{~g} \mathrm{~K}_{2} \mathrm{CO}_{3}$.

IV. $0 \cdot 2403 g$ Substanz gaben $0 \cdot 1971 g \mathrm{~K}_{2} \mathrm{PtCl}_{6}$.

In 100 Teilen:

\begin{tabular}{|c|c|c|c|c|}
\hline \multicolumn{4}{|c|}{ Gefunden } & \multirow{2}{*}{$\begin{array}{r}\begin{array}{r}\text { Berechnet für } \\
\mathrm{C}_{16} \mathrm{H}_{31} \mathrm{O}_{2} \mathrm{~K}\end{array} \\
\end{array}$} \\
\hline I. & II. & III. & IV. & \\
\hline$C \ldots \ldots \ldots 6 \cdot 34$ & - & - & - & $65 \cdot 30$ \\
\hline$H \ldots \ldots \ldots 10 \cdot 80$ & - & - & - & $10 \cdot 54$ \\
\hline $\mathrm{K} \ldots \ldots \ldots-$ & $13 \cdot 27$ & $13 \cdot 04$ & $13 \cdot 15$ & $13 \cdot 26$ \\
\hline
\end{tabular}

\section{Das Barytsalz.}

Dieses wurde auf drei Arten hergestellt:

I. aus mit Kalilauge neutralisiertèr Säure und Chlorbariumlösung. Weißes krystallinisches Pulver;

II. Säure wurde in überschüssigem Ammoniak gelöst und dann mit Chlorbarium gefälit. Krystallinisches Pulver;

III. und IV. aus freier Säure in Alkohol und essigsaurem Baryt unter Erwärmen auf dem Wasserbade. Bildet Krystallschuppen.

Die Analysen lieferten:

I. $0.2883 \mathrm{~g}$ Substanz gaben, verbrannt, $0.0883 \mathrm{~g} \mathrm{Ba} \mathrm{CO}$.

II. $0.2970 g$ Substanz gaben $0.0910 g \mathrm{BaCO}_{3}$.

III. $0.2936 \mathrm{~g}$ Substanz gaben $0.0894 \mathrm{~g} \mathrm{BaCO}$.

IV. $0 \cdot 2343 \mathrm{~g}$ Substanz gaben, mit Kupferoxyd und Kaliumbichromat verbrannt, $0.5079 \mathrm{~g} \mathrm{CO}_{2}$ und $0.2030 \mathrm{~g} \mathrm{H}_{2} \mathrm{O}$. 
In 100 Teilen:

\begin{tabular}{|c|c|c|c|c|c|}
\hline & \multicolumn{4}{|c|}{ Gefunden } & \multirow{2}{*}{$\begin{array}{l}\text { Berechnet für } \\
\mathrm{C}_{32} \mathrm{H}_{62} \mathrm{O}_{4} \mathrm{Ba}\end{array}$} \\
\hline & 1. & II. & III. & IV. & \\
\hline $\mathrm{C} \ldots \ldots \ldots$ & - & - & - & $59 \cdot 11$ & $59 \cdot 31$ \\
\hline $\mathrm{H} \ldots \ldots \ldots$ & - & $\therefore$ & - & $9 \cdot 60$ & $9 \cdot 57$ \\
\hline$\ldots \ldots$ & $21 \cdot 33$ & $21 \cdot 31$ & $21 \cdot 19$ & - & $21 \cdot 22$ \\
\hline
\end{tabular}

\section{Das Silbersalz.}

Zu mit Natronlauge neutralisierter Säure wurde ein Überschuß von salpetersaurem Silber gegeben. Der entstandene Niederschlag wurde abgesaugt und nach dem Waschen getrocknet. Das Salz stellt ein weißes amorphes Pulver dar, ist sehr leicht und wird beim Reiben stark elektrisch. Vom Tageslicht wird es nicht geschwärzt. ${ }^{1}$ Es ist löslich in heißem Toluol und fällt daraus schon bei geringer Abkühlung als Gallerte aus. Nach dem Trocknen bildet der Niederschlag mikroskopisch-kleine verfilzte Nadeln.

Bestimmungen des Silbers gaben:

I. $0 \cdot 2993 \mathrm{~g}$ Substanz lieferten nach dem Verbrennen $0.0888 \mathrm{~g} \mathrm{Ag}$.

II. $0.3040 \mathrm{~g}$ Substanz lieferten $0.0898 \mathrm{~g} \mathrm{Ag}$.

III. $0 \cdot 2464 \mathrm{~g}$ Substanz lieferten $0 \cdot 0732 \mathrm{~g} \mathrm{Ag}$.

IV. $0.2885 \mathrm{~g}$ Substanz lieferten $0.0864 \mathrm{~g} \mathrm{Ag}$.

In 100 Teilen:

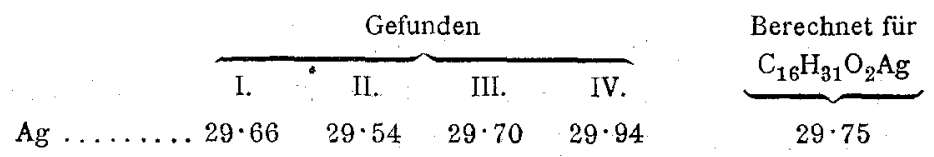

Die Elementaranalysen ergaben folgende Werte:

I. $0.2231 \mathrm{~g}$ Substanz gaben $0.4282 \mathrm{~g} \mathrm{CO} 2$ und $0.1707 g \mathrm{H}_{2} \mathrm{O}$.

II. $0.2115 \mathrm{~g}$ Substanz gaben $0.4099 \mathrm{~g} \mathrm{CO}_{2}$ und $0.1692 \mathrm{~g} \mathrm{H}_{2} \mathrm{O}$.

In 100 Teilen:

\begin{tabular}{|c|c|c|}
\hline \multicolumn{2}{|c|}{ Gefunden } & \multirow{2}{*}{$\begin{array}{l}\text { Berechnet für } \\
\mathrm{C}_{16} \mathrm{H}_{31} \mathrm{O}_{2} \mathrm{Ag}\end{array}$} \\
\hline I. & II. & \\
\hline $\mathrm{C} \ldots \ldots \ldots 52 \cdot 35$ & $52 \cdot 86$ & $52 \cdot 89$ \\
\hline$\ldots .8 \cdot 47$ & $8 \cdot 88$ & $8 \cdot 54$ \\
\hline
\end{tabular}

1 Ebenso Heintz, Ann. d. Chemie u. Pharm., 88, 299. 
Außerdem war auch das Natronsalz, das Kalksalz und das Kupfersalz hergestellt worden, von denen die durchgeführten Bestimmungen der Metalle mit den betreffenden Salzen der Palmitinsäure zusammenstimmten. Vom Kupfersalz wäre zu erwähnen, daß sich selbes in Benzol ziemlich gut beim Erwärmen löst und beim Erkalten als krystallinischer Niederschlag ausfällt.

Auch wurden der Methyl- und der Äthylester noch hergestellt, von denen zum Schlusse einiges gesagt sein soll.

\section{Der Methylester.}

Es wurden $4 \mathrm{~g}$ Säure und $30 \mathrm{~g}$ wasserfreier Holzgeist verwendet. In diese Lösung wurde $\mathrm{HCl}$-Gas bis zur Sättigung eingeleitet. Darauf wurde die Masse in Wasser gegossen, wobei der Ester sich oben als Kuchen abscheidet. Ohne besondere Trocknung wurde der Ester später im luftverdünnten Raume destilliert. Dabei siedet er bei $12 \mathrm{~mm}$ Druck bei $184^{\circ}$. Die Ausbeute betrug $3 \cdot 3 g$ von $3 \cdot 8 g$ Rohprodukt.

Der Methylester bildet eine weiße krystallinische Masse mit schwachem Geruch und hat einen Schmelzpunkt von $29^{\circ}$ Angegeben findet sich $28^{\circ} .{ }^{1}$

Eine Verbrennungsanalyse lieferte folgende Daten:

$0.2303 \mathrm{~g}$ Substanz gaben $0.6357 \mathrm{~g} \mathrm{CO}_{2}$ und $0.2580 \mathrm{~g} \cdot \mathrm{H}_{2} \mathrm{O}$.

In 100 Teilen:

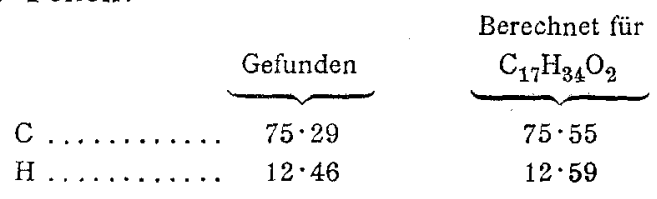

\section{Der Äthylester.}

Es wurden $5 \mathrm{~g}$ Säure und $30 \mathrm{~cm}^{3}$ absoluter Alkohol genommen und im übrigen wie beim Methylester verfahren. Der abgeschiedene Kuchen wurde hier in Äther gelöst und mit $\mathrm{CaCl}_{2}$ behandelt, bevor die Vakuumdestillation ausgeführt

1 Beilstein, 1. c. 
wurde. Sein Siedepunkt bei $10 \mathrm{~mm}$ Druck ist $191^{\circ}$. Angegeben ist 184.5 bis $185.5^{\circ}$ bei $10 \mathrm{~mm} .^{1}$

Die Rohausbeute an Ester betrug $5 g$, reiner Ester wurden $4.3 \mathrm{~g}$ erhalten. Er bildet wie der Methylester eine rein weiße krystallinische Masse, die bei $25^{\circ}$ schmilzt und dabei einen schwachen, nicht unangenehmen Geruch hat. Die Angaben des Schmelzpunktes lauten auf $24 \cdot 2$ und $24 \cdot 0^{\circ} .^{2}$

Eine Verseifung des Esters lieferte folgendes Resultat:

$0.5997 g$ Substanz brauchten zur Verseifung $0.1186 g$ Kalihydrat.

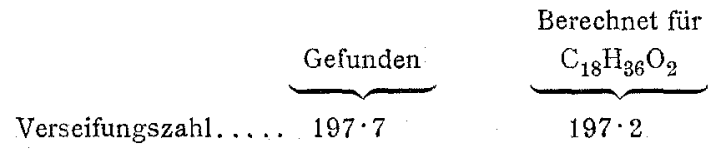

Eine Elementaranalyse ergab:

$0.2250 g$ Substanz gaben $0.6250 \mathrm{~g} \mathrm{CO}_{2}$ und $0.2632 \mathrm{~g} \mathrm{H}_{2} \mathrm{O}$.

In 100 Teilen:

\begin{tabular}{|c|c|c|}
\hline & Gefunden & $\begin{array}{l}\text { Berechnet für } \\
\mathrm{C}_{18} \mathrm{H}_{36} \mathrm{O}_{2}\end{array}$ \\
\hline & $75 \cdot 78$ & $76 \cdot 05$ \\
\hline & $12 \cdot 97$ & $12 \cdot 68$ \\
\hline
\end{tabular}

Über die anderen Zersetzungsprodukte des Balanophorins bei der Destillation im Vakuum und bei der Kalischmelze soll dann ein weiterer Teil dieser Arbeit handeln.

1 1. Ergänzungsband zu Beilstein's Handb., 1. c.

2 Ibidem und Beilstein's Handb., 1. c. 